\title{
INCLUSIÓN DE ANIMALES NO-HUMANOS EN UN MARCOO DE ARGUMENTACIÓN TEÓRICO CONTRACTUAL ${ }^{1}$
}

Daniel Loewe*

RESUMO - $O$ artigo investiga a possibilidade de justificar direitos dos animais dentro de um marco de argumentação contratualista. De acordo com a tese defendida, esta justificação é possível se a teoria contratualista requerida se distingue das tradicionais e se modifica nos modos relevantes.

PALAVRAS-CHAVE - direitos dos animais, imparcialidade, interesse, teorias contratualistas.
ABSTRACT - The article investigates the possibility of justifying animal rights within a contractarian framework of argumentation. According to the thesis developed in this paper, this justification is possible if the underlying contractarian theory is distinguished from the traditional theories and modified in relevant ways.

KEY WORDS - animal rights, contractarian theories, impartiality, interest.

No hay moral sin comunidad moral. ¿Pero quién pertenece a ella? En las diferentes teorías morales encontramos elementos estructurales que permiten contestar esta pregunta de diferentes modos. Aun cuando la esfera de la moral no se deja cubrir con la de la justicia, la situación en el caso de las teorías de justicia es similar: las arquitecturas de justificación respectivas determinan quien pertenece a la comunidad de los sujetos de justicia, esto es, quien puede formular una demanda basada en razones de justicia.

Teorías contractuales tienen una larga tradición en la filosofía política y encuentran en la actualidad amplia aceptación - por cierto, no sin objeciones relevantes. En general, se acepta que particularmente estas teorías tienen dificultades en relación a la inclusión de animales no-humanos en la esfera de la

* Forschungsstelle Politische Philosophie; Interfakultäres Zentrum für Ethik in den Wissenschaften Eberhard-Karls Universität Tübingen.

1 Versiones anteriores de este texto fueron presentadas como ponencia en la conferencia "Ergebnistagung des Graduiertenkollegs Bioethik" en el Interfakultäres Zentrum für Ethik in den Wissenschaften de la universidad Tübingen en noviembre del 2006 y en la Forschungsstelle Politische Philosophie de la Universidad Tübingen en enero del 2007. Aprovecho la ocasión para agradecer a los participantes en las discusiones.

n. 1

março 2008 
moral o de la justicia ${ }^{2}$. En este punto están de acuerdo tanto defensores de los derechos de los animales (como, por ejemplo, Regan 1984, 163-74), como también los críticos de éstos (compare, por ejemplo, Carruthers 1992). Regularmente se parte del supuesto que la ampliación de la teoría - desde humanos hasta incluir animales como sujetos de moral o de justicia - en razón de la estructura teórica contractual es imposible. La reciprocidad que yace a la base de las diversas versiones de estas teorías tornaría esta extensión problemática. Como lo formula Patzig de un modo conciso pero expresivo: "Los animales no pertenecen a los socios del contrato basado en reciprocidad que yace a la base lo la moral humana" (Patzig 1983, 14) ${ }^{3}$. Esto se aplica también a la versión quizás más refinada de estas teorías: la teoría de la justicia de Rawls.

En este artículo indagaré las posibilidades que se ofrecen desde una perspectiva teórica contractual para llevar a cabo la mencionada inclusión de los animales. En mi opinión, y contra un punto de vista dominante, teorías contractuales pueden ofrecer un marco teórico que torna posible esta inclusión. Sin embargo, las teorías contractuales apropiadas para este cometido se distinguen de las teorías contractuales clásicas de un modo relevante, que es preciso investigar. (1) En primer lugar, considero en que sentido las teorías contractuales pueden incluir animales y en que sentido - relevante para esta investigación -, no. (2) En segundo lugar, diferencio entre teorías contractuales que se basan (a) en el interés propio, (b) en la imparcialidad y (c) teorías compuestas que se basan en elementos tanto de (a) como de (b) (la teoría de Rawls). (3) Y en tercer lugar, examino la posibilidad de superar las dificultades de esta inclusión dentro de un marco argumentativo contractual. Para esto discuto sucintamente la teoría articulada Rowlands.

\section{Teorías contractuales y la inclusión de los animales}

Hay múltiples versiones de teorías contractuales, y éstas compiten entre si. Sin embargo, es posible identificar una idea fundamental: en un estado de naturaleza, esto es, en un estado caracterizado por relaciones anteriores a su positivación jurídica, los individuos lograrían un acuerdo sobre los principios de organización política que permitiesen abandonar este estado. Este acuerdo toma la forma de un contrato obligante, en razón del cual se legitima el orden político. Por cierto no se postula la existencia histórica del contrato, sino sólo su existencia hipotética. De este modo, los elementos contrafácticos juegan desde un comienzo un rol importante dentro de cualquier estructura de justificación teórica contractual. Esto es, argumentaciones del tipo: si $X$ fuese el caso, entonces se decidiría $Y$; o en una versión retroactiva: si $X$ hubiese sido el caso, se hubiese decidido $Y$. La determinación de $\mathrm{X}$ corresponde a la descripción correspondiente del estado de naturaleza, y la determinación de $\mathrm{Y}$

2 Debido a razones de concisión en vez de utilizar las expresiones compuestas "animales no-humanos" y "animales humanos" me referiré respectivamente a "animales" y a "humanos", sin implicar en este uso lingüístico una diferencia fundamental entre estos.

3 Traducciones DL 
corresponde a las reglas o principios que se escogerían en el estado de naturaleza así caracterizado.

Ya que los principios acordados y legitimados mediante un contrato en el estado de naturaleza organizan y legitiman la vida política o jurídica conjunta fuera del estado de naturaleza, teorías contractuales equiparan la pregunta: "¿quién participa en la determinación de los principios?" con la pregunta "¿para quién deberían valer estos principios?". Las obligaciones que se dejan retrotraer a estos principios y las demandas legítimas correspondientes, son válidas sólo en el caso de aquellos que (de un modo contrafáctico) legitiman el contrato, esto es, aquellos que pueden ser socios en el contrato. En forma tradicional se considera exclusivamente a los seres humanos - pero no a todos los seres humanos - como posibles socios contractuales.

Por cierto esto no significa que desde una perspectiva como la mencionada no se sea responsable por aquellas acciones que afectan al mundo animal o al medioambiente. Patzig, por ejemplo, aboga por una "Ética de la razón" (Patzig $1983,23)$. Esto es posible, porque la determinación del conjunto de los sujetos morales primeramente relevantes no ofrece una respuesta inmediata a la pregunta acerca del contenido de las obligaciones. Esto no se relaciona directamente con la estructura argumentativa de una teoría contractual, sino que con el concepto de "derecho", y se puede ilustrar recurriendo a un sencillo análisis de éste: "A tiene frente a B un derecho a X", puede ser traducido como: "B esta obligado frente a A, a cumplir X". En este análisis se determina el conjunto de los sujetos morales primeramente relevantes mediante la extensión de los As y Bs. Como determinación de la obligación, $\mathrm{X}$ puede contener contenidos diferentes. Por ejemplo: una teoría que establece que As y Bs sólo pueden ser seres humanos, puede por cierto reconocer que los seres humanos tienen una obligación frente a otros seres humanos - también en una perspectiva intergeneracional - o frente a si mismos, de considerar el bienestar de determinadas especies de animales o incluso de considerar la conservación de la naturaleza en general, aunque los animales o la naturaleza no tengan ningún derecho al cumplimiento de esta obligación moral o legal. Esto es lo que en la tradición (kantiana) se denomina una "obligación en vistas a" (en vez de "frente a") ${ }^{4}$.

En un cierto sentido es posible afirmar que los animales beneficiados mediante el cumplimiento de este tipo de obligaciones pueden ser considerados como portados de derechos. Ser un beneficiado del cumplimiento de la obligación de otros sin ser simultáneamente portador del derecho correspondiente, puede ser todavía caracterizado como un estatus de titular de derecho - en todo caso, no en un sentido primario, sino que únicamente en un sentido derivado. A menudo se refiere a derechos indirectos y se los diferencia de los derechos directos (compare Rowlands 1998, cap.

4 "Pflichten in Ansehung von" y "Pflichten gegenüber". 
6) para caracterizar este tipo de titulación de derechos ${ }^{5}$. A continuación esbozaré este modo de referirse a derechos de un modo conciso, para posteriormente referirme a la pregunta relativa al modo en que la inclusión de los animales es problemática para las teorías contractuales.

Derechos directos: un individuo tiene un derecho directo (o es el portador de un derecho directo) a un acción determinada o a un bien determinado, cuando (a) el individuo tiene el derecho a esa acción o bien, y (b) el tener este derecho no depende de la existencia de los derechos de otro individuo (o asociación).

Derechos indirectos: un individuo tiene un derecho indirecto (o es el portador de un derecho indirecto) a una acción determinada o a un bien determinado, cuando (a) el individuo tiene el derecho a esa acción o bien, y (b) el tener este derecho depende de la existencia de los derechos de otro individuo (o asociación).

La existencia de derechos indirectos se puede fundar mediante variados tipos de argumentos. A continuación consideraré de un modo conciso dos formas de argumentación:

Primero: el tipo de relación que tengo hacia mi perro puede ser legalmente protegida mediante el reconocimiento de mis derechos (directos). El caso más conocido y sencillo refiere al reconocimiento del derecho de propiedad: en este caso, Usted no debe dañar mi propiedad (mi perro), porque de otro modo perjudicaría mis derechos y así mis variados intereses protegidos por éstos. Pero el tipo de relación hacia los animales legalmente protegida mediante derechos (directos) puede se concebida de un modo más amplio que el que refiere exclusivamente a los derechos de propiedad: por ejemplo, mis derechos, los derechos de los otros, o los derechos de las generaciones futuras a un mundo animal diverso, pueden fundar protección legal (bajo la condición que los argumentos a favor de la existencia de esos derechos sean convincentes).

Segundo: una estructura de argumentación muy conocida se articula de acuerdo a la siguiente estrategia: tratar a un animal de un modo cruel es moralmente reprobable, pero no en razón del daño que afecta al objeto de la acción cruel (el animal), sino que en razón de las consecuencias dañinas que la acción cruel produce en su causante (este es el famoso "argumento del embrutecimiento" que Kant articula en favor de las "obligaciones en vistas a" ${ }^{6}$ ). Estas consecuencias tornan más probable

5 En todo caso la atribución de derechos directos a la naturaleza no-sintiente o a artefactos no es posible: ya que ellos no pueden tener intereses en sentido estricto (ellos no pueden tener actitudes proposicionales), ellos no pueden -a diferencia de muchos animales-ser un sujeto lógico de derechos, tampoco de derechos indirectos. (A menos, por cierto, que la referencia a "derechos indirectos" sea exclusivamente un modo de hablar. Pero en ese caso se podría afirmar que también tractores y aparatos fotográficos tienen derechos indirectos -lo que parece ser una consecuencia poco plausible de ese modo de hablar que, en todo caso, induce a errores). A diferencia de lo recién mencionado, una "obligación en vistas a" puede prohibir o ordenar acciones en relación a la naturaleza no-sintiente o a artefactos. Lo que diferencia los derechos indirectos de los directos, es que los intereses del portador del primer tipo de derechos no son comprendidos inmediatamente en la argumentación moral, sino sólo de un modo mediado: porque ellos son relevantes para el portador del segundo tipo de derechos.

6 Este es el así denominado: "Verrohungsargument". 
que aquel que trata animales de un modo cruel, tratará también a los seres humanos de un modo cruel. En el caso de estos (y otros) argumentos parece ser apropiado utilizar la forma lingüística que el animal es portador de derechos indirectos, cuya existencia se basa en derechos directos de otros (seres humanos o asociaciones).

En principio, estos argumentos son compatibles con un marco de argumentación teórico contractual. Los intereses compartidos que son protegidos al asegurar los derechos de propiedad (para así, por ejemplo, asegurar un marco que permita el desarrollo de la autonomía individual), o al conservar ciertos bienes particulares también para el futuro -, o el interés en el cumplimiento de la ley moral, pueden ser comprendidos dentro de un marco de argumentación teórico contractual como razones para establecer una obligación mutua ${ }^{7}$. De este modo, se puede argumentar dentro de un marco de argumentación teórico contractual a favor de la existencia de derechos indirectos (en vez de directos). Correspondientemente, sería posible finalizar esta investigación con la afirmación que, en un cierto sentido (esto es: mediante obligaciones en vistas a, o mediante derechos indirectos), un marco de argumentación teórico contractual hace posible la inclusión de los animales y sus intereses y necesidades particulares en la comunidad moral. Pero, ¿es satisfactoria esta forma relacional?

En mi opinión, esta forma relacional es insatisfactoria porque no se considera a los animales como sujetos morales primarios. Ellos lo son exclusivamente en un sentido derivado. La consideración de los animales, con sus intereses y necesidades particulares, es aquí indirecta. Ella se funda o en el interés propio de los seres humanos o en las (supuestas) obligaciones de los seres humanos hacia si mismos (como en el caso de la teoría de Kant) o hacia otros seres humanos (también en una perspectiva intergeneracional). Pero la afirmación que se es un sujeto moral en un sentido derivado no tiene sentido. $O$ se es un sujeto moral, o no. Esto no implica prejuzgar una respuesta a la pregunta acerca de qué es lo que debería implicar la toma en consideración de este estatus. Esto depende de las teorías particulares, y ciertamente hay buenas razones para fundar una protección diferenciada en razón de la consideración del sujeto moral. Pero en tanto los intereses y necesidades de los animales no sean directamente considerados, los animales no serán reconocidos en ningún sentido razonable como sujetos morales primarios (que pueden ser sujetos de justicia) con demandas morales legítimas que deben ser consideradas.

Esta dificultad de las teorías contractuales para incluir animales como sujetos morales o de justicia primarios, está estrechamente relacionada con momentos estructurales de estas teorías. Por una parte esto se relaciona con el momento motivacional en la teoría (esto es, las razones para acceder a realizar un contrato con los otros). Por otra parte esto se relaciona con un concepto de persona que quizás

7 Un uso ejemplar de esta posibilidad de argumentación se encuentra, por ejemplo, en la estrategia desarrollado por Wissenburg para fundar la protección del medioambiente en el marco de la teoría de la justicia de Rawls recurriendo al principio de ahorro justo (compare Wissenburg 1998). 
es necesario para la estructura de las teorías contractuales. A continuación examinaré estos dos elementos centrales para las teorías contractuales, pero problemáticos para la inclusión de los animales en la esfera de la justicia ${ }^{8}$.

\section{Ventajas mutuas y capacidades vinculadas a la razón}

De un modo tradicional es posible identificar una diferenciación entre dos corrientes teóricas contractuales (compare Barry 1989, 1995; Kymlicka 1990): hay teorías contractuales que se basan en el interés propio, y teorías contractuales que se basan en una concepción de imparcialidad. Ambas formas de contractualismo reconocen que los seres humanos, o al menos la mayoría de los seres humanos, son iguales por naturaleza. Pero ellas tienen diferentes concepciones acerca de la referencia de esta igualdad. Una tercera posibilidad toma la forma de una teoría compuesta (compare Nussbaum 2006).

\subsection{Promoción de los intereses propios mediante el afianzamiento de ventajas mutuas}

El primer grupo de teorías está muy próximo a las teorías contractuales clásicas: ellas se basan en la promoción de los intereses propios mediante el afianzamiento de ventajas mutuas. Una característica central de estas teorías es el momento motivacional: porque los individuos quieren promover sus intereses propios, o tantos de éstos como sea posible, están motivados a abandonar el estado de naturaleza mediante el cierre de un contrato que, al asegurar los intereses mutuos promueve los intereses propios. "Intereses" son entendidos en un sentido estrecho como nuestros fines privados y no como fines ajenos considerados en razón de motivos altruistas ${ }^{9}$. Un conocido representante de una posición de este tipo, que se puede considerar como un ejemplo paradigmático, es David Gauthier con su libro Moral by Agreement (1986), o en la literatura en lengua alemana Peter Stemmer con su libro Handeln zugunsten anderer (2000). La respuesta a la pregunta: "¿porqué debo asentir a la relación contractual?", es simultáneamente motivante: porque corresponde a tu interés propio. Negarse a asentir el contrato sería un signo de irracionalidad entendida de un modo estrecho: se persigue un fin, pero se rechazan los medios que conducen a éste. La gran ventaja de esta forma de argumentación es quizás su equipaje moral ligero: la justificación de los principios legitimados mediante un contrato en el estado de naturaleza se realiza sin apelar a un motivo moral o a un valor disputado y encuentra, por lo tanto, una amplia posibilidad de aceptación. De este modo - en caso que funcione - esta argumentación gana en fuerza justificativa.

8 En la tercera parte de este artículo examinaré la posibilidad de fundar derechos directos de animales dentro de un marco teórico contractual.

9 Dentro de los elementos teóricos contractuales en la teoría de derecho natural articulada por Locke tiene la benevolencia una gran importancia. Pero, a pesar de esto, los individuos son motivados porque mediante el contrato ellos pueden promover mejor sus intereses: "for their comfortable, safe, and peaceable living amongst another, in a secure Enjoyment of their properties, and a greater Security against any that are not of it" (Locke 1960 (1679), capítulo 8, §95). 
La condición de esta forma de argumentación, es que el contrato y el orden que éste legitima, efectivamente promuevan los intereses propios. Si esto es así o no, está estrechamente relacionado con la caracterización del estado de naturaleza realizada por las teorías particulares. Por ejemplo, si tengo buenas razones para suponer que soy suficientemente fuerte para imponer siempre mis intereses en el estado de naturaleza, sin arriesgar que su promoción se vea frustrada por las acciones concertadas de los otros, mi motivación para abandonar el estado de naturaleza será mínima. Es por esto que las teorías contractuales se dedican en forma intensiva a la descripción del estado de naturaleza. Éste es caracterizado - con mayor o menor dramatismo - mediante escasez de bienes, mediante el conflicto y mediante una potencialidad de poder similar entre los individuos. Aunque las descripciones del estado de naturaleza son diferentes, se encuentra un mínimo común múltiplo: no se puede ser tan poderoso como para poder decidir cualquier conflicto a favor del propio bien. Mediante el aseguramiento de ventajas mutuas, el cierre de un contrato debe implicar un rendimiento positivo para cada socio.

La inclusión de los animales y sus intereses en un marco argumentativo como el expuesto colisiona rápidamente contra barreras insuperables: para ganar la motivación de los individuos en el estado de naturaleza para aceptar el contrato, ellos no pueden estar en la situación de poder imponer siempre sus intereses en el estado de naturaleza. Esto implica que en el estado de naturaleza ellos deben tener una potencialidad de poder más o menos similar, lo que estas teorías explícitamente acentúan ${ }^{10}$. Esta tesis puede ser en el caso de los seres humanos una premisa aceptable - sobre todo si se considera la posibilidad de acciones concertadas. Pero en el caso de los animales esta tesis es ciertamente inverosímil: la diferencia de poder entre seres humanos y animales es demasiado grande. Correspondientemente, las ventajas que los seres humanos pueden anticipar en razón de un contrato con éstos son demasiado pequeñas para que de este modo valiese la pena reconocer a los animales como socios.

Por lo demás, esto no es válido exclusivamente en el caso de los animales, sino que también en el caso de seres humanos con deficiencias físicas y mentales importantes. Por ejemplo, de acuerdo a Gauthier la inclusión de seres humanos con deficiencias físicas y mentales en la esfera de la justicia es, debido a la razón mencionada, extremadamente difícil o incluso imposible. El estado de naturaleza debe ser una ficción coherente. Esto implica una apreciación realista de los poderes, de las necesidades y de la relación fundamental hacia los otros socios del contrato. Si los únicos argumentos válidos para llevar a cabo un contrato se relacionan con la promoción de los intereses propios racionales, no disponemos de ninguna razón para entender el conjunto de los socios del contrato de un modo más amplio al que es necesario para poder promoverlos.

10 Hobbes, por ejemplo, a quien usualmente se recurre como un representante clásico de esta forma de contractualismo, acentúa la igualdad de poder de la mayoría de los seres humanos, la cual se debe a su naturaleza. 


\subsection{Imparcialidad como premisa fundamental}

El segundo grupo de teorías contractuales está compuesto por aquellas que se basan en el valor de la imparcialidad ${ }^{11}$. La respuesta a la pregunta: "iporqué debo asentir a la relación contractual?", no es primeramente una apelación a la promoción de los intereses propios, sino que una apelación a nuestro interés moral de establecer una relación moral justificable con y hacia los otros. Dicho de otro modo: estas teorías dan por sentado el motivo moral. De este modo, ellas cargan un equipaje moral más pesado que las teorías arriba mencionadas. La igualdad, que las teorías contractuales que se basan en el interés propio caracterizan como igualdad natural en relación a la vulnerabilidad y la capacidad de poder, es entendida por estas teorías como una igualdad moral que en cada caso debe ser considerada: podemos promover nuestros intereses, bajo la condición que en este proceso reconozcamos a los otros como sujetos morales relevantes y los respetemos en forma correspondiente. Con palabras kantianas (y estas teorías son a menudo denominadas teorías de inspiración kantiana (compare Kymlicka 1991)), esto significaría no tratar a los otros simplemente como medio, sino que siempre simultáneamente como fin. Pero estas teorías contractuales no proporcionan ninguna justificación de esta igualdad moral. Lo que ellas ofrecen es una aclaración de lo que esta igualdad fundamental debiera implicar.

En sus diversas interpretaciones, la imparcialidad implica que debemos una justificación a todos aquellos que son afectados en razón de nuestro accionar, y una justificación tal, que ellos - como sujetos morales relevantes - puedan aceptar. Así, el acceso para participar en el contrato no está mediado por el potencial de amenaza de cada cual, sino que por esta igualdad moral que debe ser respetada. Esta justificación debida toma en ocasiones (pero no siempre) la forma de un consentimiento general. Por ejemplo, de acuerdo a Kant una norma moral es justificada, cuando cada cual la puede querer como una ley general.

Problemático para la inclusión de los animales en este marco argumentativo, es que la posibilidad de esta justificación adeudada sólo es válida entre entidades que poseen determinadas capacidades. El concepto de persona, en tanto refiere a un sujeto que debe ser considerado en forma moral, es definido a menudo en razón de estas capacidades ${ }^{12}$. En el caso de las teorías contractuales es usual remitirse a la capacidad de poder asentir o rechazar la justificación adeudada, y también de poder actuar de acuerdo a las reglas justificadas mediante el contrato. Pero esta capacidad para asentir y para actuar implica ciertas capacidades morales que, tradicionalmente, sólo son asignadas a los seres humanos. De este modo, el reconocimiento de los animales en tanto socios del contrato y la consideración de sus intereses y necesidades parece ser imposible desde un comienzo: ya que los animales

11 Ejemplos de teorías contractuales de carácter político y moral que se basan en la imparcialidad son las articuladas por Barry $(1989,1995)$ y Scanlon $(1998)$ respectivamente.

12 Una pregunta importante, es si estas capacidades son necesarias o sólo suficientes para la definición del concepto de persona. En la próxima sección retornaré a este punto. 
(y ciertamente la naturaleza no-sintiente) no son seres autónomos, no pueden se considerados en forma moral a un nivel primario de la teoría.

\subsection{Una teoría compuesta: La Teoría de la Justicia de Rawls}

La teoría de la justicia de Rawls, como es articulada en A Theory of Justice y en Political Liberalism, es actualmente no sólo la más conocida, sino que probablemente la teoría contractual más finamente articulada. En esta teoría se encuentran elementos de ambas tradiciones. Hasta que punto estos elementos se complementan o conducen a una tensión basal interna en la teoría, es una pregunta difícil de responder a la que no me referiré en este texto. Para esta investigación basta con mostrar que en la teoría de Rawls se encuentran las dificultades señaladas relacionadas con la inclusión de los animales ${ }^{13}$.

Rawls imagina una nueva versión del estado de naturaleza, que ahora está caracterizado por un mayor nivel de abstracción: la así denominada posición original (original position). Mediante un experimento mental cualquiera puede situarse en la posición original. Ésta es caracterizada mediante el así denominado "velo de la ignorancia". Éste pone límites estrechos a los conocimientos disponibles: no disponemos de conocimientos particulares sobre nosotros mismos. Sabemos que tenemos una concepción del bien, pero no sabemos cuál. Tampoco sabemos cuál es nuestra posición social y económica, esto es: cuan privilegiados o desaventajados somos en comparación social. Los participantes en la posición original suponen, que ellos siempre disponen sobre un determinado mínimo de capacidades físicas y mentales, pero ellos no saben cuan dotados ellos son por sobre este mínimo. Ellos saben que en la sociedad están vigentes lo que Hume denominó las "circunstancias de la justicia", esto es: escasez relativa de recursos y la generosidad limitada de la naturaleza humana. En un contexto como el expuesto, los individuos deben escoger principios de justicia en razón de los cuales deben ser organizadas las instituciones políticas más importantes de la sociedad en la que ellos van a vivir. Los individuos no son impulsados en esta elección por envidia, sino que por el intento de ganar para si tantas ventajas como sea posible. Ellos no son impulsados por una motivación moral, sino que por sus intereses propios racionales. Pero ya que en razón del velo de la ignorancia ellos no saben quienes son en la sociedad (su concepción del bien, su fuerza física, talentos y su posición económica y social), no pueden preferir principios que los privilegien de un modo especial. Al escoger principios que avancen sus intereses, escogen simultáneamente principios que privilegien a cualquiera que se encuentre en su situación de elección. De este modo, la posición original representa una situación de imparcialidad, y las decisiones tomadas en ésta son justas (fair). Rawls afirma que una decisión en la posición original corresponde a una decisión benévola fuera de ésta.

13 Aunque la pregunta relativa a la relación de los dos textos mencionados de Rawls es atingente y necesaria (las interpretaciones de esta relación van desde una relación de complementariedad hasta una de incompatibilidad), yo los trataré como una unidad. En mi opinión, del modo en que se estructure esa relación no resulta ninguna diferencia relevante para los fines argumentativos de este texto. 
La intuición fundamental de Rawls - o como él dice: "uno de los puntos fijos de nuestros juicios meditados" (Rawls 1971, 104) - está también a la base de las teorías liberales igualitarias (pero no a la base de todas las teorías liberales): tanto nuestro punto de partida social y económico, como también nuestra dotación de talentos son "contingentes" y por lo tanto "moralmente arbitrarias" (arbitrary from a moral point of view). Ellos son resultado de la lotería natural. Nadie puede afirmar que merece su dotación de talentos innatos o su posición económica y social inicial en la sociedad. "Merecer" supone la existencia de un esquema de cooperación social. Rawls amplia el campo de validez de esa idea incluso a la posesión de un carácter superior que trae consigo la iniciativa para la formación de capacidades. Con las palabras de Rawls:

Parece ser uno de los puntos fijos de nuestros juicios meditados que nadie merece su lugar en la distribución de dotes innatos más de lo que uno merece su lugar de partida inicial en la sociedad. La afirmación que un individuo merece su carácter superior que posibilita que se esfuerce en el cultivo de sus habilidades es igualmente problemática; porque su carácter depende en gran medida de circunstancias familiares y sociales afortunadas por las que él no puede reclamar crédito (Rawls 1971, 104)

Pero estos elementos moralmente arbitrarios tienen consecuencias importantes en las oportunidades en la vida de cada cual. Para poder garantizar igualdad de oportunidades estas consecuencias deben ser reguladas mediante principios de justicia. Sólo cuando las instituciones más importantes de la sociedad se organizan de acuerdo a estos principios es posible tener "expectativas legítimas" sobre los frutos de nuestro obrar (es decir: merecerlos).

La teoría de Rawls tiene las mismas dificultades que fueron identificadas en los dos tipos de teorías contractuales. Por una parte Rawls entiende la sociedad como una empresa cooperativa. En concordancia con las teorías contractuales clásicas Rawls afirma que los individuos son "rough equals", esto es: que ellos disponen por sobre un mínimo de capacidades físicas y mentales. Debido a las circunstancias de la justicia los "rough equals" adquieren la motivación para realizar un contrato, y de este modo asegurar las ventajas mutuas. Pero si ellos están por debajo del mínimo estipulado de capacidades físicas y mentales, no habría ninguna razón para reconocerlos como socios en el contrato social: las ventajas anticipadas de la cooperación serían demasiado mínimas. No es casual que la teoría de Rawls (en forma similar a la teoría de Gauthier) no tienen lugar para seres humanos con deficiencias mentales y físicas considerables al nivel primario de la teoría, esto es: al nivel en el que se deciden los principios de la justicia ${ }^{14}$. El caso de los animales es aun más claro: ya que los animales no son "rough equals", esto es: su capacidad de cooperación y de poder es mínima, no habría en la teoría ningún lugar para ellos como socios del contrato. Por consiguiente los animales no pueden ser sujetos de justicia.

14 Martha Nussbaum ha criticado recientemente de un modo convincente que en la teoría de justicia de Rawls los seres humanos con discapacidades físicas y mentales no son reconocidos como sujetos de justicia (Nussbaum 2006). 
Por otra parte Rawls hace uso en la posición original de un concepto (kantiano) de persona, que se caracteriza mediante los "two moral powers". Personas morales son definidas en razón de la posesión de estos poderes morales. Ellos implican (a) la capacidad para formar una concepción del bien, y (b) un sentido de la justicia, esto es: un deseo efectivo para emplear principios de justicia y actuar de acuerdo a éstos. La igualdad moral a la base de las teorías contractuales que se basan en la imparcialidad se define en la teoría de Rawls mediante el concepto de persona caracterizado mediante los dos poderes morales. Y de acuerdo a Rawls, son las personas morales así definidas, las únicas que pueden ser sujetos de justicia: "La respuesta natural parece ser que son precisamente las personas morales quienes tienen un derecho a justicia igual" (Rawls 1971, 505). Resulta evidente que los animales no poseen estos poderes morales. Por lo tanto ellos no serían ni personas morales, ni sujetos de justicia, y correspondientemente no pueden ser socios en el contrato.

Esta interpretación es quizás apresurada. Rawls no afirma en ningún lugar de su obra que la capacidad para ser persona moral es necesaria para poder tener una demanda de justicia. De acuerdo a Rawls: "Nosotros reconocemos entonces, que la capacidad para la personalidad moral es una condición suficiente para tener un derecho a justicia igual. Nada más allá de este mínimo esencial es requerido. Yo dejo de lado si la personalidad moral es también una condición necesaria" (Rawls 1971, 505-6). La teoría de Rawls deja por lo tanto una posibilidad abierta: eventualmente ciertos seres que no disponen de los poderes morales podrían ser sujetos de justicia. ¿Implica lo anterior que también los animales podrían ser sujetos de justicia?

En la teoría de Rawls no se encuentra ninguna reflexión que pudiese sustentar esta tesis. En vez de esto, Rawls descarta explícitamente esta posibilidad: "yo no he afirmado que la capacidad de un sentido de la justicia sea necesaria en orden a que le debamos [a un ser] obligaciones de justicia, pero de todos modos parece que no estamos requeridos para ejercer justicia estricta hacia seres a los que les falta esta capacidad" (Rawls 1971, 512). Rawls acentúa explícitamente que en nuestro trato con los animales tenemos "obligaciones de compasión" y "obligaciones de humanidad": "La capacidad por de sentir placer y dolor y las formas de vida de las que los animales son capaces claramente imponen obligaciones de compasión y de humanidad en su caso" (Rawls 1971, 512). Estas no son, sin embargo, obligaciones de justicia ${ }^{15}$.

15 De acuerdo a Rawls, aunque los animales están presumiblemente excluidos del campo de validez de los principios de la justicia: "ellos tienen por cierto alguna protección, pero su estatus no es el de los seres humanos" (Rawls 1971, 505). Pero aquí hay un error: aunque Rawls afirma que la mayoría de los seres humanos disponen al menos por sobre un cierto mínimo sobre estas capacidades, también hay seres humanos que claramente no cumplen con esta condición. Correspondientemente no sólo animales, sino que también ciertos seres humanos son excluidos como socios del contrato: de este modo, la integración de seres humanos con discapacidades mentales considerables (y que por tanto no disponen de los dos poderes morales) como socios del contrato sería de acuerdo a su teoría imposible (compare Nussbaum 2006). 
Si bien Rawls deja abierta la posibilidad que se pudiese tener obligaciones de justicia también frente a ciertos seres que no disponen de las dos capacidades morales, es difícil imaginar cómo se podría fundar esta posibilidad en un marco de argumentación teórico contractual rawlsiano.

\section{Extensión de un marco de argumentación teórico contractual}

Si estamos de acuerdo con los análisis hasta aquí esbozados, tenemos dos posibilidades: o no se le atribuye a los animales un estatus moral en tanto personas morales y correspondientemente en tanto sujetos de justicia, o se renuncia a uno de los marcos de argumentación actualmente de uso más corriente y quizás uno de los más productivos: las teorías contractuales. Ambas posibilidades son poco atractivas. Por lo tanto hay que someter otra posibilidad a discusión: ¿es posible extender un marco de argumentación teórico contractual de tal modo que los animales con sus intereses y necesidades particulares sean incluidos a un nivel primario de la teoría? A continuación indagaré esta posibilidad.

\subsection{Como animal en la posición original}

Imagine que está en la posición original, pero - entre otras cosas - Usted no sabe si es un animal o un ser humano: ¿qué principios escogerían para organizar el mundo?

Esta pregunta marca el punto de partida de una posible extensión de la teoría contractual. Esta idea fue expresada ya muy temprano por Hare en relación a la teoría de justicia de Rawls: "Evidentemente es difícil incluir animales entre la POPs [people in the original position - DL] (ellos no pueden llevar a cabo discursos en la asamblea); pero este es un efecto desafortunado del escenario dramático - que quizás podría ser superado si nosotros pudiésemos suponer que la POPs no saben si ellos no serán o llegarán a ser animales" (Hare 1975, 97; el artículo original fue publicado en 1973). También Wenz trabaja esta idea en Environmental Justice (1988). Y una elaboración detallada de esta idea se encuentra en los libros de Mark Rowlands Animal Rights: A Philosophical Defence (1998) y Animals Like Us (2002). A continuación examinaré algunos de los aspectos de la teoría elaborada por Rowlands para así indagar la posibilidad de esta extensión.

Rowlands pretende integrar los intereses y necesidades de los animales al primer nivel de la teoría contractual de Rawls - esto es: al nivel en que se escogen los principios de la justicia. De acuerdo a Rowlands, en el marco argumentativo de la teoría de justicia de Rawls sería posible justificar derechos (directos) de los animales. Todavía más: al considerar la intuición liberal fundamental en la cual se basa la teoría de Rawls (compare 2.3), para mantener la coherencia de la teoría deberíamos otorgar derechos a los animales. El rechazo explícito de Rawls de esta posibilidad se debería a que él no seguiría consecuentemente las implicaciones de la intuición fundamental de su teoría hasta el final.

Sin embargo, en mi opinión no es evidente que esta extensión de la teoría de Rawls sea posible. Si bien Rowlands reconoce que las diferencias entre su 
contractualismo y el de Rawls son grandes, y que habría que hablar por tanto de una nueva teoría rawlsiana, no es claro que estas diferencias no sean tan fundamentales que la extensión de la teoría de Rawls propuesta por Rowlands vaya más allá de lo que el marco teórico de la teoría de Rawls permite.

Primero: A diferencia de Rawls, quien con su teoría contractual aspira a formular una concepción de justicia social, esto es: una concepción de justicia cuyo objeto son las instituciones sociales más importantes, Rowlands aspira con su teoría contractual a fundar una concepción moral, esto es: principios morales que regulen las relaciones entre los sujetos relevantes.

Segundo: A diferencia de la teoría de Rawls, que como ha sido examinado incorpora elementos de las dos tradiciones contractuales, esto es el interés propio y la imparcialidad, la teoría contractual propuesta por Rowlands se basa exclusivamente en la imparcialidad. Esto queda claramente establecido en el uso conceptual de Rowlands, quien denomina el contexto de elección original (esto es: el estado de naturaleza de las teorías contractuales clásicas) "impartial position" y no, como Rawls, "original position".

Estas son diferencias fundamentales entre las dos teorías. La primera se refiere al foco de la teoría y la segunda a su base normativa. Es difícil pensar como un cambió de elementos tan centrales para el marco argumentativo rawlsiano puede ser entendido como una extensión de su teoría. Como mencione, la tesis de Rowlands es todavía más radical: él sostiene que la formulación teórica que él presenta no es sólo una extensión de la teoría de Rawls, sino que correspondería a la interpretación correcta de la intuición a la base de su teoría. Pero si, como vimos, ya la extensión puede ser puesta en cuestión, es aún más claro que esta es una interpretación incorrecta de la teoría de Rawls.

Sin embargo, si el fin argumentativo es integrar los intereses y las necesidades particulares de los animales al primer nivel de una teoría contractual (y no el dar cuenta de la interpretación correcta de la teoría de Rawls, o el intentar extenderla de acuerdo a los requerimientos de la coherencia argumentativa interna de la teoría), es la caracterización de la teoría propuesta por Rowlands razonable y productiva. El uso de una teoría basada en la imparcialidad (y no en el interés propio, o en una mezcla de ambos) es crucial para llevar a cabo la inclusión de los intereses y las necesidades particulares de los animales en un marco argumentativo contractual. A continuación me referiré a este punto:

Primero: resulta evidente que una teoría contractual en que se recurre al interés propio como el motor de la justificación, y correspondientemente se propone alguna noción de igualdad en la potencialidad de poder, como, por ejemplo, la concepción de "rough equality" propuesta por Rawls, no permite la inclusión de los animales. La idea de una "rough equality" entre seres humanos y animales no es sostenible: las diferencias en el potencial de poder son demasiado grandes. Si queremos protegernos del potencial de poder de los animales, o si queremos asegurarnos ventajas en nuestro modo de tratar a los animales, y así promover nuestros intereses propios racionales, entonces no hay ninguna razón para aceptarlos como interlocutores o socios en el contrato. En vez de esto hay buenas razones para seguir 
tratándolos como lo hacemos y correspondientemente seguir instrumentalizándolos para nuestros fines.

Segundo: teorías contractuales que recurren al interés propio como motor de su justificación son en general poco satisfactorias. Al situar la capacidad de poder en un lugar central de la teoría, simultáneamente establecen criterios y mecanismos de exclusión arbitrarios. Muchas personas parecen no tener mayores dificultades cuando esta exclusión refiere a los animales - lo que posiblemente se deja retrotraer a tradiciones de tratamiento de animales históricamente arraigadas. Sin embargo, con el fin de examinar críticamente este elemento excluyente realice el siguiente experimento mental: imagine que una comunidad de extraterrestres decide invadir la tierra. Sus capacidades mentales, y también la potencialidad de poder que han desarrollado, están en una relación hacia nuestras capacidades mentales y nuestra potencialidad de poder similar a aquella, en la que nuestras capacidades mentales y potencialidad de poder están en relación a las de una vaca. Todavía más: los extraterrestres consideran que los seres humanos son criaturas repugnantes, pero que, apropiadamente aderezados y cocinados, son un plato exquisito. Sin embargo, ya que los extraterrestres son seres razonables (sus capacidades mentales superan a las nuestras, como las de nosotros superan a las de una vaca), en vez de simplemente invadir la tierra para disfrutar de sus recursos y espacio de vida, destruir a una parte de sus habitantes humanos y establecer criaderos, deciden invitar a un representante de los seres humanos para que les explique porqué los extraterrestres debería considerar los intereses de los seres humanos. De este modo ellos invitan a un filósofo (seres sumamente racionales) que sostiene una teoría contractual que se basa en la promoción de los intereses propios. ¿Qué tipo de argumentos podría articular este filósofo a favor de la especie humana? Lamentablemente, ya que la diferencia de poder es tan grande, no hay argumentos disponibles para incluir a los seres humanos en la comunidad moral o de sujetos de justicia. Por sobre esto, ya que los extraterrestres tienen una aversión hacia los seres humanos, no parecen haber razones que permitan justificar derechos indirectos, como, por ejemplo, en el caso de las mascotas (quedan disponibles los argumentos que se refieren al derecho (directo) de propiedad de los extraterrestres sobre los humanos, lo que por cierto no es demasiado prometedor). De este modo, la única posibilidad disponible es apelar a la compasión de los extraterrestres.

\subsection{La arbitrariedad de la pertenencia a una especie}

En cada teoría contractual es central la caracterización del estado de naturaleza. Este es también el caso de la posición original en la teoría de Rawls. Como Rawls afirma, primero debemos saber que descripción del estado de naturaleza aceptamos - después de todo, hay una descripción apropiada de ésta para obtener diferentes principios. De acuerdo a Rawls, una de las razones para aceptar una descripción particular del estado de naturaleza es que ésta conduzca a los principios que intuitivamente consideramos aceptables. Y nosotros los consideramos aceptables, porque ellos corresponden a nuestros juicios bien-meditados. En el proceso de caracterización del estado de naturaleza trabajamos desde los dos fines ("work from 
both ends"), esto es: cuando los principios de justicia definidos mediante una determinada caracterización del estado de naturaleza colisionan con nuestros juicios bien-meditados, podemos (a) revisar nuestros juicios, o (b) caracterizar nuevamente el estado de naturaleza. Este es el así denominado equilibrio reflexivo.

De acuerdo a la intuición (igualitaria) fundamental de Rawls: cuando una propiedad no es merecida, en el sentido que no se puede considerar al individuo como responsable por poseerla, ella es moralmente arbitraria, y por lo tanto no se tiene ningún derecho (no-cualificado) a las ventajas que se dejan remitir a ella en un proceso de cooperación social. Esa intuición fundamental juega un rol central, cuando se trata de considerar como arbitrarias la posición inicial social y económica o las dotaciones naturales innatas. Correspondientemente, el estado de naturaleza es definido de tal modo que los participantes no puedan tener acceso a la información correspondiente. La pregunta más importante en la caracterización del estado de naturaleza refiere al tipo de información sobre la que los socios del contrato no deben poder disponer. De este modo es posible excluir lo moralmente irrelevante de la argumentación.

Rowlands argumenta que la pertenencia a una especie es irrelevante para la argumentación moral que se desarrolla en su posición imparcial. Esto se deduciría directamente de la intuición fundamental en la que se basa la teoría de justicia de Rawls. El estatus de la pertenencia a una especie no sería distinguible del estatus de nuestra posición social y económica en la sociedad. La pertenencia a una especie puede ser ciertamente remitida a la lotería natural, es decir, ella es moralmente arbitraria: no podemos hacer nada por o contra ella y no la merecemos. De acuerdo a Rowlands, este argumento torna la pertenencia a una especie irrelevante en tanto información para tomar una decisión moralmente obligante en el estado de naturaleza. En este sentido, ella debe ser tratada en forma análoga a nuestra situación social o económica inicial o a nuestras capacidades físicas y mentales. Así, las consecuencias que se dejan retrotraer a hechos contingentes y moralmente arbitrarios, como la pertenencia a una especie determinada, deben ser reguladas mediante principios de justicia.

Pero este argumento no va suficientemente lejos. Aunque es correcto que no podemos hacer nada por o contra nuestra pertenencia a una especie y que no la merecemos, hay que preguntar, porqué esta información debiera ser considerada como relevante en un marco de argumentación rawlsiano. La idea fundamental de Rawls, que está a la base de las teorías liberales igualitarias, es que los seres humanos deben tener oportunidades iguales. Por lo tanto, hechos contingentes sobre los que no tienen control y que influencias sus oportunidades de un modo relevante deben ser neutralizados mediante principios de justicia. El que se trata de las oportunidades de seres humanos y no de las oportunidades de los animales es en el marco de argumentación de Rawls razonable: como vimos, es la capacidad de disponer de los dos poderes morales lo que define a una persona moral y correspondientemente a un sujeto de justicia, pero los animales no tienen esas capacidades.

Rowlands intenta debilitar esta objeción - que se puede articular desde una perspectiva rawlsiana - recurriendo a la diferenciación introducida por Regan pero 
ya tradicional entre agentes morales y pacientes morales. Agentes morales son definidos mediante la capacidad de poder razonar moralmente. Para esto juegan los poderes morales de Rawls - sobre todo el sentido de la justicia - un rol importante. Pacientes morales no pueden razonar moralmente. Pero esto no implica que lo que se haga a los pacientes morales sea irrelevante. Esto se debe a que los pacientes morales tienen un bienestar, y por lo tanto, de acuerdo a Rowlands, ellos merecen consideración moral (Rowlands 2002, 63). Pero el que se sea un agente $o$ un paciente moral es también resultado de la lotería natural y por lo tanto es también un hecho contingente. De este modo también la información acerca de si somos un agente o un paciente moral no debiera estar disponible en el estado de naturaleza. Sin el conocimiento acerca de cual es nuestra especie, y sin el conocimiento acerca de si somos un agente o un paciente moral, debemos escoger principios para la organización de nuestro mundo. En un estado de naturaleza así definido, nadie preferiría principios que perjudicasen fuertemente a los pacientes morales.

La definición de "pacientes morales" ofrecida por Rowlands es: "cualquiera (o cualquier cosa) que merece consideración moral" (Rowlands 2002, 63). Pero esto es una definición de una clase (pacientes morales) mediante una propiedad normativa (los que merecen consideración moral). La pregunta interesante debiera ser: ¿porqué los pacientes morales merecen consideración moral? La respuesta de Rowlands a esta pregunta es: "un paciente moral es cualquier cosa cuyo bienestar Usted está obligado a considerar al actuar" (Rowlands 2002, 63). Esa respuesta puede ser interpretada de tres modos diferentes: la primera posibilidad, es que los agentes morales están obligados a considerar el bienestar de los pacientes morales porque deben realizar un valor moral particular en el mundo, o deben cumplir obligaciones hacia si mismos o hacia otros. Pero si este fuese el caso, no habría que incluir precisamente a los animales y sus intereses y necesidades en un marco teórico contractual. Esta interpretación correspondería a la ya mencionada forma tradicional de fundar protección mediante el cumplimiento de "obligaciones en vistas a". Esto no puede ser lo que Rowlands pretende. La segunda posibilidad es que el bienestar en cuanto tal es lo que nos obliga. A favor de esta posibilidad hay buenas razones. Sin embargo, esta interpretación se ajusta mejor a una teoría que se guía mediante el fomento del bienestar que a una teoría contractual. La tercera posibilidad constituye el núcleo de la argumentación de Rowlands: estamos obligados a considerar el bienestar de los animales porque éste podría ser nuestro bienestar. El concepto de persona, que Rawls define en razón de los dos poderes morales, debe ser definido aquí exclusivamente mediante la propiedad de poder poseer algún bienestar ${ }^{16}$.

16 Una crítica usual a este tipo de argumentación pone en cuestión que se disponga del nivel de imaginación necesario para llevar a cabo estos experimentos mentales. Pero esta crítica no da en el blanco. Si bien es correcto que la posibilidad de imaginar que se es un animal en la posición original colisiona con nuestra experiencia del mundo de un modo en que quizás no colisiona con el imaginarnos siendo otro ser humano, este tipo de imaginación no es necesario para desarrollar el experimento mental. No es -con la famosa 


\subsection{Consideraciones finales}

Siguiendo los argumentos articulados en este texto es posible afirmar, contra la opiniones dominantes, que un marco de argumentación teórico contractual puede incluir a los animales con sus intereses y necesidades particulares dentro de la esfera de la moral y la justicia. Sin embargo, este marco teórico contractual tiene características que lo diferencian de un modo relevante de la mayoría de las estructuras argumentativas contractuales a las que usualmente se recurre en la actualidad.

Este marco teórico contractual no se basa en la promoción de los intereses propios mediante el afianzamiento de ventajas mutuas, sino que encuentra sus bases en un valor independiente: la imparcialidad. Correspondientemente, esta teoría no aspira a encontrar y legitimar los principios que regulen la cooperación social así como los resultados y las cargas asociadas con ésta, sino que aspira a establecer una relación normativa hacia todas las entidades moralmente relevantes que se base en el deseo de vivir con ellos en términos sostenibles desde una perspectiva moral. Y de acuerdo a la extensión realizada de un marco argumentativo contractual, las entidades morales relevantes son todos los seres que pueden tener un bienestar (y no todos los seres que tienen los poderes morales).

Este tipo de contractualismo se aleja de un modo considerable de las teorías tradicionales, en tanto presupone la motivación de querer vivir con todos los seres que pueden tener un bienestar de un modo moralmente defendible, o dicho de otro modo, presupone la motivación de querer justificar nuestras acciones frente a los otros de un modo tal, que nosotros podamos razonablemente esperar que éstos estén dispuestos a aceptar (compare Scanlon 1998). El que los otros en determinadas ocasiones no puedan entender las justificaciones no implica que esta motivación deje de existir. La inclusión de los animales y sus intereses y necesidades particulares en este tipo de argumentación es posible bajo la condición que queremos estar en una relación hacia ellos que sea moralmente sostenible -una actitud que quizás no pocos comparten, sin estar, sin embargo, concientes de sus consecuencias.

\section{Bibliografía}

Barry, Brian: Justice as Impartiality (Oxford University Press 1995)

Barry, Brian: Theories of Justice (University of California Press 1989)

Carruthers, Peter: The Animal Issue: Moral Theory in Practice (Cambridge University Press 1992)

DeGrazia, David: Taking Animals Seriously. Mental Life and Moral Status (Cambridge University Press 1996)

fórmula de Nagel-"what it is likeness" lo que debe ser imaginado, sino sólo de que-oraciones. Y para realizar estas oraciones podemos recurrir a todo el conocimiento del que disponemos sobre el tipo de animal en cuestión. Aquí hay también por cierto límites más o menos estrechos -depende del animal en cuestión. Pero estos límites dependen de nuestros conocimientos empíricos y no de nuestra posibilidad de tener acceso a los Qualia de otro ser. 
Gauthier, David: Moral by Agreement (Oxford University Press 1986)

Hare, H. L. A.: "Rawls' Theory of Justice" En: Norman Daniels: Reading Rawls. Critical Studies of A Theory of Justice (Basil Blackwell 1975)

Kymlicka, Will: “The social contract tradition" En: Peter Singer (Ed.): A Companion of Ethics (Blackwell 1991)

Locke, John: Two Treatises of Government (Peter Laslett (Ed.) Cambridge University Press $1960(1679-80))$

Nussbaum, Martha: Frontiers of Justice. Disability, Nationality, Species Membership (Harvard University Press 2006)

Nussbaum, Martha: "Beyond "Compassion and Humanity": Justice for Nonhuman Animals" En: Sunstein/Nussbaum: Animals Rights. Current Debates and New Directions (Oxford University Press 2004)

Patzig, Günther: "Ökologische Ethik - innerhalb der Grenzen bloßer Vernunft" (Vandenhoeck \& Ruprecht 1983)

Rawls, John: A Theory of justice (Harvard University Press 1971)

Rawls, John: Political Liberalism (Columbia University Press 1993)

Regan, Tom: The Case for Animals Rights (Routledge 1984)

Rowlands, Mark: Animal Rights. A Philosophical Defence (Palgrave MacMillan 1998)

Rowlands, Mark: Animals Like Us (Verso 2002)

Scanlon, Thomas: What We Owe to Each Other (Harvard University Press 1998)

Searle, John R.: "Der Geist der Tiere" En: Perler/Wild: Der Geist der Tiere (Suhrkamp: Frankfurt am Main 2005)

Stemmer, Peter: Handeln zugunsten anderer (de Gruyter 2000)

Wenz, Peter S.: Environmental Justice (State University of New York Press 1988)

Wissenburg, Marcel: Green Liberalism. The free and the green society (Routledge 1998) 